\section{A Case of Rheumatoid Arthritis Associated with SMAD3 Gene Mutation: A New Clinical Entity?}

To the Editor:

Apart from the Marfan syndrome and the Loeys-Dietz syndromes, aneurysms may occur with mutations of the SMAD3 and are associated with early osteoarthritis $(\mathrm{OA})^{1}$. We describe a case in which a patient with the SMAD3 mutation had multiple aneurysms and rheumatoid arthritis (RA).

A 65-year-old woman had had seropositive erosive RA since 1982. Her disease was severe and in 2007 required treatment with etanercept. Hand and feet radiographs showed no signs of OA.

The patient was also being followed up for aneurysms in several medium-sized arteries. A left internal carotid artery aneurysm was embolized in 1998 and caused an ischemic stroke. She also had bilateral dysplastic aneurysms of the vertebral arteries and a chronic type III descending aorta dissection with a thoraco-abdominal aneurysm treated surgically in 1999. In 2008, she underwent revision surgery for a pseudoaneurysm at the reinsertion site of the medullary arteries and opening of the left iliac aneurysm at the cannulation site. In May 2008 she was found to have a hepatic artery aneurysm that was laid open, and a vein bypass was done to the right hepatic artery. She still has a left carotid artery aneurysm at the anastomosis with the arch of the aorta, which is monitored regularly.

She had a similar family history, her father having had aneurysms of the renal artery. Her daughter and son had aortic aneurysms.

In 2010 and 2012, she consulted a multidisciplinary team to investigate for Marfan syndrome. This diagnosis was excluded because she had only 2 minor skeletal signs, pectus excavatum and a high arched palate. Radiographs showed thoracic spine OA, posterior joint OA, and severe left coxarthrosis secondary to a coxitis.

Genetic testing was performed to look for a familial form of arterial aneurysms. Sequencing of 9 exons of the SMAD3 gene was done. In the exon 3, using the primers SMAD3-ex3F1 (CGT GAA TCC CTA CCA CTA CCA) and SMAD3-ex3R1 (AGG GAT GCG GTT CTG AGT C), a duplication of 5 nucleotides (c401_405dup) was found, leading to a heterozygous mutation (p.Pro136 Phefs*52) and introducing a premature stop codon.

Autosomal dominant mutations of the SMAD3 gene cause a syndrome combining thoracic and abdominal aneurysms with early OA of the fingers, wrist, and spine. These are associated with craniofacial features (hypertelorism and palatal or uvular abnormalities), skeletal (pectus excavatum, scoliosis, and protruded acetabuli), and skin abnormalities (striae, varicose veins, and inguinal and umbilical hernias) ${ }^{2,3}$.

The syndrome has a high mortality rate because of a high risk of aneurysmal dissection and rupture ${ }^{1}$. Most of the SMAD3 mutations are located in a region of the gene, the $\mathrm{MH} 2$ domain, which is highly preserved in all species. These result in dysregulation of the transmission of transforming growth factor (TGF)- $\beta$ in extracellular matrix cells and lead to increased expression in aortic expression of several key players of the TGF- $\beta$ pathway, including phosphorylation of SMAD2 and SMAD3 proteins, upstream ligands such as TGF- $\beta 1$ and downstream targets such as collagen or connective tissue growth factors ${ }^{2}$. Dysregulation of TGF- $\beta$ appears to cause histological disorganization of the media, with fragmentation and loss of elastic fibers and collagen accumulation in the media.

Dysregulation of the TGF- $\beta$ signaling pathways is seen in other aneurysmal diseases including Marfan syndrome, owing to a mutation of the FBN1 gene, and Loeys-Dietz syndrome, through a mutation of the TGFB2 gene $^{4}$.

SMAD3 gene mutations are also involved in genetic predispositions to $\mathrm{OA}^{5}$. The TGF- $\beta$ pathway is involved in phosphorylation of SMAD2 and SMAD3 molecules, which help to maintain the typical phenotype of joint chondrocytes.

TGF- $\beta$ is a key cytokine in immune system regulation. Targeting T lymphocytes and the role of the TGF- $\beta$ pathway in the development of some autoimmune diseases is well described, such as in systemic sclerosis ${ }^{6}$. The role of TGF- $\beta$ in RA is uncertain; it may have both proinflammatory and antiinflammatory activity. In vitro, TGF- $\beta 1$ injection into the joints of healthy rats causes joint inflammation and destruction. Local or systemic injections of antibodies that neutralize TGF- $\beta 1$ in mice that are predisposed to develop collagen-induced arthritis increase the severity of the arthritis ${ }^{7}$.

TGF- $\beta$ is expressed by synovial tissue and is found in the synovial fluid of patients who have RA ${ }^{7}$. Increased expression of TGF- $\beta$, type 1 and type 2 TGF- $\beta$ receptors, and endoglin is reported ${ }^{8}$. This increase in TGF- $\beta$ correlates positively with clinical markers of disease activity, and there appears to be a link between the development of inflammation and activation of the TGF- $\beta$ signaling pathway. This may provide a potential underlying explanation for the development of RA as a result of a SMAD3 gene mutation.

To our knowledge, this is the first case report in the literature of a $S M A D 3$ gene mutation associated with arterial aneurysm dissections and RA. If confirmed, this association may broaden the clinical spectrum of this syndrome.

ELISE BERTHET, MD, Centre Hospitalier Universitaire (CHU) Clermont Ferrand, Rheumatology, Clermont Ferrand; NADINE HANNA, MD, AP-HP Hospital Bichat, Paris; CHARLOTTE GIRAUD, MD; MARTIN SOUBRIER, PhD, CHU Clermont Ferrand, Rheumatology, Clermont Ferrand, France. Address correspondence to Dr. M. Soubrier, Service de Rhumatologie, Hôpital Gabriel Montpied, 63003 Clermont Ferrand, France.E-mail: msoubrier@chu-clermontferrand.fr

\section{REFERENCES}

1. van der Linde D, van de Laar IM, Bertoli-Avella AM, Oldenburg RA, Bekkers JA, Mattace-Raso FU, et al. Aggressive cardiovascular phenotype of aneurysms-osteoarthritis syndrome caused by pathogenic SMAD3 variants. J Am Coll Cardiol 2012;60:397-403.

2. van de Laar IM, Oldenburg RA, Pals G, Roos-Hesselink JW, de Graaf BM, Verhagen JM, et al. Mutations in SMAD3 cause a syndromic form of aortic aneurysms and dissections with early-onset osteoarthritis. Nat Genet 2011;43:121-6.

3. van de Laar IM, van der Linde D, Oei EH, Bos PK, Bessems JH, Bierma-Zeinstra SM, et al. Phenotypic spectrum of the SMAD3-related aneurysms-osteoarthritis syndrome. J Med Genet 2012;49:47-57.

4. Gomez D, Al Haj Zen A, Borges LF, Philippe M, Gutierrez PS, Jondeau G, et al. Syndromic and non-syndromic aneurysms of the human ascending aorta share activation of the Smad2 pathway. J Pathol 2009;218:131-42.

5. Valdes AM, Spector TD, Tamm A, Kisand K, Doherty SA, Dennison EM, et al. Genetic variation in the SMAD3 gene is associated with hip and knee osteoarthritis. Arthritis Rheum 2010;62:2347-52.

6. Granel B, Chevillard C, Dessein A. [Genetic factors predisposing to fibrosis in systemic sclerosis]. [Review in French] Rev Med Interne 2005;26:294-303.

7. Gonzalo-Gil E, Criado G, Santiago B, Dotor J, Pablos JL, Galindo $\mathrm{M}$. Transforming growth factor (TGF)- $\beta$ signalling is increased in rheumatoid synovium but TGF- $\beta$ blockade does not modify experimental arthritis. Clin Exp Immunol 2013;174:245-55.

8. Pohlers D, Beyer A, Koczan D, Wilhelm T, Thiesen HJ, Kinne RW Constitutive upregulation of the transforming growth factor-beta pathway in rheumatoid arthritis synovial fibroblasts. Arthritis Res Ther 2007;9:R59.

J Rheumatol 2015;42:3; doi:10.3899/jrheum.140645 